\title{
NOVO MODELO DE TELETRIAGEM DAS CARDIOPATIAS CONGÊNITAS
}

\author{
Tele-screening New Model of Congenital Heart Disease \\ Lúcia Roberta Didier Nunes Moser; Tereza Cristina Pinheiro Diogenes²; Vanessa Oliveira Pacífico de Souza ${ }^{3}$; \\ Alyne Ranaci Florêncio de Oliveira ${ }^{4}$; Felipe Alves Mourato5; Sandra da Silva Mattos ${ }^{6}$
}

Resumo Um modelo de triagem das cardiopatias congênitas foi criado associando a telemedicina com a captação de imagens ecocardiográficas básicas por neonatologistas. Foi baseado num treinamento presencial e na orientação dos casos via internet. Esse modelo é viável, desde que sob tutela de um cardiologista pediátrico, pois reduz custos e distâncias no contexto do diagnóstico das cardiopatias congênitas.

Palavras-chave: Telemedicina, Ecocardiografia, Triagem Neonatal, Cardiopatias Congênitas

\begin{abstract}
A "tele-screening" model for CHD was developed using telemedicine coupled with online training of neonatologists on the acquisition of basic cardiac views. An initial live training session was provided and subsequently the examinations were oriented online. Under cardiology supervision, the tele-screening model proved to be reliable as it reduced cost and distances in the context of congenital heart disease. Keywords: Telemedicine, Neonatal Screening, Congenital Heart Defects, Echocardiography
\end{abstract}

\footnotetext{
1. Coordenadora Clínica da Rede de Cardiologia Pediátrica - Médica especialista em cardiologia pediátrica; 2. Médica plantonista da Rede de Cardiologia Pediátrica - Médica especialista em ecocardiografia e cardiologia pediátrica; 3. Médica plantonista da Rede de Cardiologia Pediátrica - Médica especialista em cardiologia pediátrica; 4. Plantonista da Rede de Cardiologia Pediátrica - Médica especialista em cardiologia pediátrica; 5. Coordenador Científico da Rede de Cardiologia Pediátrica - Graduado em Medicina; 6. Coordenadora Geral da Rede de Cardiologia Pediátrica - Doutorado em Biotecnologia.
} 


\section{Introdução}

As cardiopatias congênitas são um dos principais motivos de morbimortalidade neonatal ${ }^{1}$, atingindo aproximadamente 8 a cada 1000 nascidos vivos ${ }^{2}$. Elas agrupam um conjunto variado de malformações cardíacas estruturais, com apresentações clínicas distintas. Algumas delas, ditas críticas, necessitam de diagnóstico ainda nos primeiros dias de vida, devido a sua rápida evolução para condições ameaçadoras da vida.

O exame de escolha para o diagnóstico das cardiopatias congênitas é o ecocardiograma transtorácico realizado por cardiologista pediátrico. Entretanto, geralmente esses profissionais estão concentrados nas grandes cidades ${ }^{3}$, dificultando o diagnóstico precoce em regiões mais isoladas.

Dessa forma, foi montado um novo modelo de triagem das cardiopatias congênitas no estado da Paraíba. Este modelo é baseado na adição de competências ao neonatologista, com orientação de cardiologistas pediátricos via telemedicina.

\section{Relato da Experiência}

A presente experiência foi realizada no estado da Paraíba, no nordeste brasileiro. Sua estrutura de saúde é composta por duas "ilhas" com serviços de alta complexidade e vastas regiões isoladas com precário acesso a especialistas.

Nesse contexto foi criada uma rede de cardiologia pediátrica com um centro de referência localizado na capital do estado vizinho, Pernambuco. Essa rede é baseada na triagem das cardiopatias congênitas por meio da oximetria de pulso arterial com auxílio de especialista via telemedicina em 13 centros espalhados pelo estado da Paraíba.
Entretanto, o único local onde há presença de cardiologistas pediátricos é na capital, o que inviabiliza a transferência constante de pacientes para esse local para realização do ecocardiograma.

Com isso em mente, foi elaborado um novo modelo para triagem das cardiopatias congênitas. Neonatologistas foram convidados a realizarem um treinamento no centro de referência em Pernambuco. Esse treinamento consistia em um curso teórico-prático com os seguintes pontos: noções de ultrassom, anatomia cardíaca, hemodinâmica e cortes ecocardiográficos necessários para uma triagem ecocardiográfica de patologias cardíacas que coloquem em risco a vida do neonato após alta hospitalar. Os cortes ecocardiográficos selecionados foram: imagem de quatro câmaras, das vias de entrada e saída dos ventrículos nos eixos longo e curto paraesternal.

Após o curso, os neonatologistas iniciaram os ecocardiogramas nos seus respectivos centros. Tais centros possuíam um tablet e conexão à internet banda larga, que serviam como ponte para o centro de referência em cardiologia pediátrica. Neste, havia cardiologistas pediátricos de plantão diurno para orientar na obtenção das imagens e, caso necessário, das condutas a serem tomadas com o paciente. Caso o ecocardiograma feito por neonatologistas exigisse maior detalhamento, o paciente era transferido para João Pessoa ou diretamente para o centro de referência, dependendo da gravidade do caso. Caso o ecocardiograma ocorresse num momento onde cardiologistas pediátricos estivessem indisponíveis, os mesmos podiam ser gravados num sistema online e posteriormente analisados.

\section{Discussão}

O morbimortalidade das cardiopatias congênitas, assim como o processo do seu diagnóstico, é um 
problema mundial. Isso se torna ainda mais grave em países subdesenvolvidos, onde o déficit de profissionais capacitados e dificuldades estruturais nos serviços de saúde são uma constante. Adicionalmente, o fato de os cardiologistas pediátricos estarem concentrados nos grandes centros urbanos resulta num grande número de neonatos descobertos. Nesse contexto, a adição de competências para outros profissionais é essencial para uma triagem adequada no contexto atual. A oximetria de pulso arterial é um bom método de triagem ${ }^{4}$ que pode, inclusive, ser realizado por profissionais de enfermagem. Entretanto, tal método não tem a capacidade de excluir ou confirmar a presença de cardiopatias congênitas, nem de orientar a conduta inicial frente a um caso positivo.

Dessa forma, a inserção do neonatologista nesse fluxo diagnóstico é vantajosa. Além de terem mais experiência com o exame físico e processos fisiopatológicos nos neonatos, também são responsáveis pelas altas hospitalares dos mesmos. Logo, a capacitação de tais profissionais para realização de um ecocardiograma de triagem com apoio de cardiologistas pediátricos via telemedicina só tem adicionar.

\section{Comentários Finais}

A adoção de modelos de triagem para cardiopatias congênitas é essencial frente ao cenário atual. A adição de competências a enfermagem (oximetria de pulso) e neonatologistas (ecocardiograma de triagem) com orientação de cardiologistas pediátricos via telemedicina mostra-se uma opção vantajosa.

\section{Referências}

1. Hoffman JIE, Kaplan S. The incidence of congenital heart disease. J Am Coll Cardiol. 2002 Jun;39(12):1890-900

2. Tandon A, Sengupta S. Risk Factors for Congenital Heart Disease CHD in Vellore, India. Curr Res J. 2010;2(4):253-8.

3. Carvalho CL, Petta HL, Araújo JF, Girardi Junior JB, Oliveira V de A. Avaliação nacional da demanda de médicos especialistas percebida pelos gestores de saúde. Belo Horizonte: Universidade Federal de Minas Gerais; 2009.

4. Ewer AK, Middleton LJ, Furmston AT, Bhoyar A, Daniels JP, Thangaratinam S, et al. Pulse oximetry screening for congenital heart defects in newborn infants (PulseOx): a test accuracy study. Lancet. 2011 Aug 27; 378 (9793): 785-94. 